\title{
Transcanal Microscopic vs Endoscopic Type I Tympanoplasty in Dry Central Perforation: A Comparative Retrospective Study
}

\author{
Ravneet $\mathrm{R}_{\text {Verma }}{ }^{-}$, Ravinder Verma $^{2}$ (C)
}

\begin{abstract}
Introduction: Type I tympanoplasty or myringoplasty is the repair of the perforated eardrum. Comparisons of microscopic and endoscopic techniques present in the literature use different routes of access and cannot be compared head-on.

Objective: To compare endoscopic and microscopic myringoplasty results when both are performed via the transcanal route.

Methods: We present a retrospective study done at a single center to compare endoscopic and microscopic type I tympanoplasties both done via the transcanal route. A total of 70 patients, 30 operated with endoscope and 40 with microscope, were included in the analysis. Patients of either sex, between 18 and 60 years of age, with a dry perforation and air-bone gap (ABG) of $\leq 30 \mathrm{~dB}$, were included.

Results: Perforations were completely closed in $93 \%$ of the endoscopic group and $92.5 \%$ of the microscopic group. The ABG closure was $12.89 \mathrm{~dB}$ in endoscopic and $11.97 \mathrm{~dB}$ in the microscopic groups. There was no association of the site or size of perforation with failure of surgery. The time taken for surgery was also equivalent. The endoscope had the advantage of avoiding a canaloplasty and looking into the hidden areas of the middle ear.

Conclusion: For transcanal, minimally invasive type I tympanoplasty, both techniques provide equivalent results. We recommend the use of an endoscope as the primary or an accessory tool for better visualization.

Keywords: Endoscopic approach, Microscope, Myringoplasty, Tympanoplasty.

Otorhinolaryngology Clinics: An International Journal (2021): 10.5005/jp-journals-10003-1364
\end{abstract}

\section{INTRODUCTION}

The aim of tympanoplasty is to eradicate the disease from the middle ear and reconstruct the hearing mechanism. Wullstein in 1956 had classified tympanoplasty into five types. Type I tympanoplasty or myringoplasty involves the repair of the perforated tympanic membrane when the middle ear chain is intact. It can be performed via endaural, postaural, or transcanal routes, and the graft can be placed by overlay, interlay, or inlay techniques.

Microscope has been the workhorse of all otological surgeries and is still the preferred visualization tool for most surgeons. Microscopic ear surgery enables two-handed manipulation and binocular vision along with an excellent stereoscopic surgical view. With limited visual access to the hidden areas, such as the sinus tympani, hypotympanum, and epitympanum, the use of a microscope in the transcanal approach is limited and a postauricular incision is preferred to obtain a wider view.

Endoscope-assisted microscopic surgery has been developed since El Guindy ${ }^{1}$ reported 36 cases of endoscopic myringoplasty and McKennan ${ }^{2}$ introduced endoscopy for second-look ear surgery. Thomassin et al. ${ }^{3}$ had used an endoscope as an adjuvant in microscopic ear surgery.

Transcanal approach has the distinct advantage of a much smaller or hidden incision, minimum soft tissue dissection, and shorter duration of surgery. Since its advent, the endoscope has transformed the external auditory canal into the operative gateway. Transcanal endoscopic ear surgery permits a magnified, wide-angle vision at a high resolution, as well as the direct visualization of hidden areas.
${ }^{1}$ Department of ENT, Head and Neck Surgery, GMCH, Chandigarh, India ${ }^{2}$ Department of Otolaryngology, Verma Hospital and Research Centre, Jalandhar, Punjab, India

Corresponding Author: Ravinder Verma, Department of Otolaryngology, Verma Hospital and Research Centre, Jalandhar, Punjab, India, Phone: +91 9814064395, e-mail: verma1999@gmail.com How to cite this article: Verma RR, Verma R. Transcanal Microscopic vs Endoscopic Type I Tympanoplasty in Dry Central Perforation: A Comparative Retrospective Study. Int J Otorhinolaryngol Clin 2021;13(1):7-10.

Source of support: Nil

Conflict of interest: None

Previous studies have compared results of microscopic surgery done via endaural or postaural routes to endoscopic surgery via transcanal route. ${ }^{1-7}$ In this study, by performing both endoscopic and microscopic myringoplasties via transcanal route, a symmetrical comparison was possible. The two arms were compared on the success of graft uptake, hearing improvement and time duration of surgery. An analysis on the resulting outcome with both techniques in relation to the size and site of the perforation was also done.

\section{Materials and Methods}

Seventy patients were selected with single, central perforation and history of the dry ear without medication for a minimum duration of 1 month. Patients in the age bracket of 18-60 years were included. Air-bone gap ( $A B G$ ) of $\leq 30 \mathrm{~dB}$ and sensorineural hearing (bone 
conduction) loss $\leq 20 \mathrm{~dB}$ on pure tone audiometry (PTA) were set as hearing threshold limits. Patients with retraction pockets, adhesive or atrophic process, subtotal perforations, extensive tympanosclerosis, and traumatic perforations were excluded. The patients with a history of otogenic complications and active external ear canal disease, exostosis, or extremely narrow tortuous ear canals where a transcanal approach was not possible were also excluded.

All patients were evaluated in terms of history, examination, and relevant investigations. Ears were examined for side, size, site, and margins of perforation, middle ear mucosal status, and aural discharge. All patients were counseled regarding the procedure and anesthesia. Informed consent was taken. PTA for hearing level and impedance test for tubal patency was performed in all patients before surgery.

The perforation size was recorded at the time of surgery. The largest diameter was used to grade the perforations into small $(<3 \mathrm{~mm})$, medium $(3-5)$, and large $(>5 \mathrm{~mm})$. The perforation sites were also categorized according to the predominant area involved as anterior, posterior, and inferior. Perforation of any size, which involved more than one segment, was classified as mixed.

Zeiss OPMI microscope and 2.9 and $4 \mathrm{~mm} 0^{\circ}$ endoscopes without a holding device, were used. Thirty-degree scopes were occasionally used to inspect the middle ear cavity in endoscopic surgeries.

All the surgeries were performed under local anesthesia, within the established principles of otologic surgery. The temporalis fascia graft was harvested with a temporal incision along the hairline. To harvest the tragal perichondrium, a curvilinear incision was made on the medial aspect of the tragus, removing the cartilage from its bed, and harvesting the perichondrium from both sides. The cartilage was then placed back. The grafts were dried and cut into the required shape and size. Next, the site and size of perforation were recorded. The margins of perforation were freshened. Tympanomeatal flap was elevated, starting 3-4 mm lateral to the annulus and going $270^{\circ}-360^{\circ}$ around the canal. The middle ear was examined for any pathology and ossicular continuity, and mobility was confirmed. The graft was placed and tucked under the handle of the malleus. After repositioning the tympanomeatal flap, gel foam pieces followed by a medicated aural pack were placed. The graft incisions were sutured. Pressure dressing was applied where temporalis fascia was harvested. The duration of the surgery was recorded. Aural pack was removed after 10 days. Ear examination was performed at each postoperative visit, and hearing assessment was done after 3 months.

\section{Results}

The transcanal microscopic myringoplasty (TMM) and the transcanal endoscopic myringoplasty (TEM) groups were comparable on age and sex of the patients, size, and site of perforation (Table 1). More than two-thirds of patients were men. Age distribution was quite even; $47 \%$ were aged 35 years or less and the rest were older. The average age in both the groups was between 36 and 37 years of age. On examination, medium-sized perforations ( $3-5 \mathrm{~mm}$ in greatest dimension) were most seen. Perforations located anteriorly and those of mixed variety were the most common according to the site.

Temporalis facial graft was more commonly used in TMM, while tragal perichondrium use was more common in TEM, but there was no significant difference ( $p$-value $=0.19$ ) (Table 2 ). In the initial part of the study, using temporalis fascia for TMM was the norm, which accounts for the higher number of patients in that category.
The duration of surgery was calculated from the time of local infiltration to aural packing. A little over half of all surgeries in both groups were completed within 60 minutes (Table 3).

The success of the surgery was defined on two parameters, the rate of closure of perforation and the improvement in $A B G$. The perforation closure had similar results in both groups (Fig. 1). There was no association of the site or size of perforation with the failure rate. The ABG closure rate was $12.89 \mathrm{~dB}$ in the TEM group, which was higher than the TMM group at $11.97 \mathrm{~dB}$, but the difference was not significant (Fig. 1).

None of our patients had preoperative or postoperative complications. Since all the patients were operated on day care basis, there were no differences in hospital stay or resource consumption. The postoperative management was similar in all patients.

The surgeons were well versed with the microscopic techniques, and in the initial phase, there was some difficulty with an endoscope, predominantly due to the availability of just one hand for instrumentation. There was also a tendency to select wider and straighter ear canals for the endoscopic procedures. For these reasons, the first few cases operated with the endoscope were not included in the study.

The endoscope allowed an unobstructed view of the tympanic membrane (Fig. 2) and had a clear advantage while inspecting the middle ear (Fig. 3). In comparison, the microscopic view is limited (Fig. 4) and difficult to assess the middle ear (Fig. 5). Angled

Table 1: Comparison of demographic factors and perforation characteristics

\begin{tabular}{|c|c|c|c|c|}
\hline \multicolumn{2}{|l|}{ Demographic factors } & $\begin{array}{c}\text { TMM } \\
(n=40)\end{array}$ & $\begin{array}{c}\text { TEM } \\
(n=30)\end{array}$ & $\begin{array}{c}\text { Total } \\
(n=70)\end{array}$ \\
\hline \multirow[t]{2}{*}{ Sex } & Female & 12 & 10 & $22(31.4 \%)$ \\
\hline & Male & 28 & 20 & 48 (68.6\%) \\
\hline \multirow[t]{2}{*}{ Age } & $\leq 35$ years & 19 & 14 & $33(47.1 \%)$ \\
\hline & $>35$ years & 21 & 16 & 37 (52.9\%) \\
\hline \multicolumn{2}{|c|}{ Average age (in years) } & 36.07 & 36.93 & 36.44 \\
\hline \multirow[t]{2}{*}{ Side } & Left & 15 & 16 & $31(44.3 \%)$ \\
\hline & Right & 25 & 14 & $39(55.7 \%)$ \\
\hline \multirow[t]{3}{*}{ Perforation size } & Small & 9 & 4 & $13(18.6 \%)$ \\
\hline & Medium & 19 & 15 & $34(48.6 \%)$ \\
\hline & Large & 12 & 11 & $23(32.8 \%)$ \\
\hline \multirow[t]{4}{*}{ Site of perforation } & Anterior & 14 & 12 & $26(37.1 \%)$ \\
\hline & Posterior & 12 & 6 & $18(25.7 \%)$ \\
\hline & Inferior & 3 & 2 & $5(7.1 \%)$ \\
\hline & Mixed & 11 & 10 & 21 (30\%) \\
\hline
\end{tabular}

Table 2: Type of graft used

\begin{tabular}{lllll}
\hline Graft material & Temporalis fascia & 25 & 14 & 39 \\
& Tragal perichondrium & 15 & 16 & 31 \\
\hline
\end{tabular}

Table 3: Comparison of duration of surgery between the two groups

\begin{tabular}{lccc}
\hline Duration of surgery & $T M M$ & TEM & Total \\
\hline Up to 60 minutes & $22(55 \%)$ & $16(53.33 \%)$ & 38 \\
60-90 minutes & $15(37.5 \%)$ & $13(43.33 \%)$ & 28 \\
$>90$ minutes & $3(7.5 \%)$ & $1(3.33 \%)$ & 4 \\
\hline
\end{tabular}




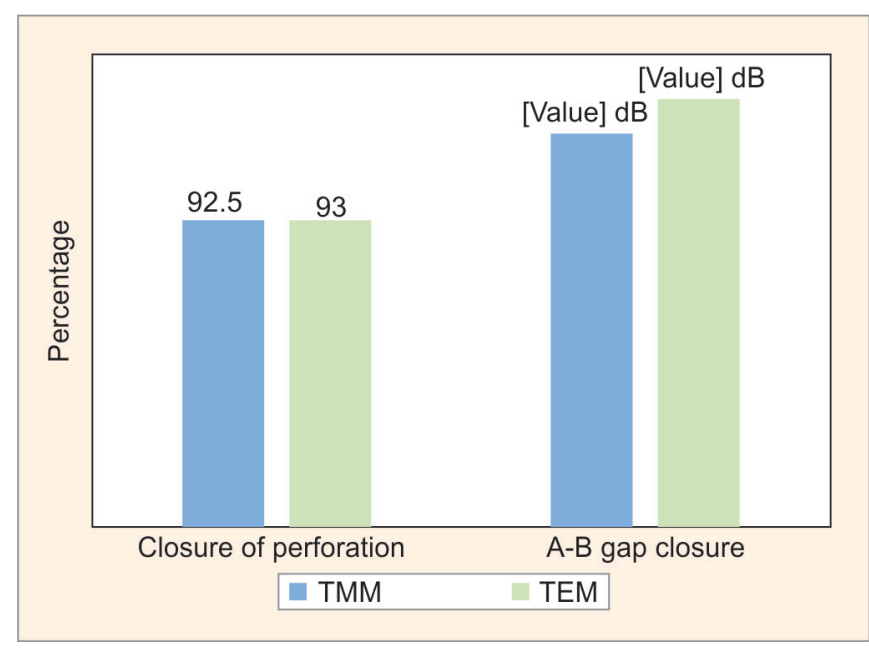

Fig. 1: Comparison of success in the closure of perforation and improvement of hearing

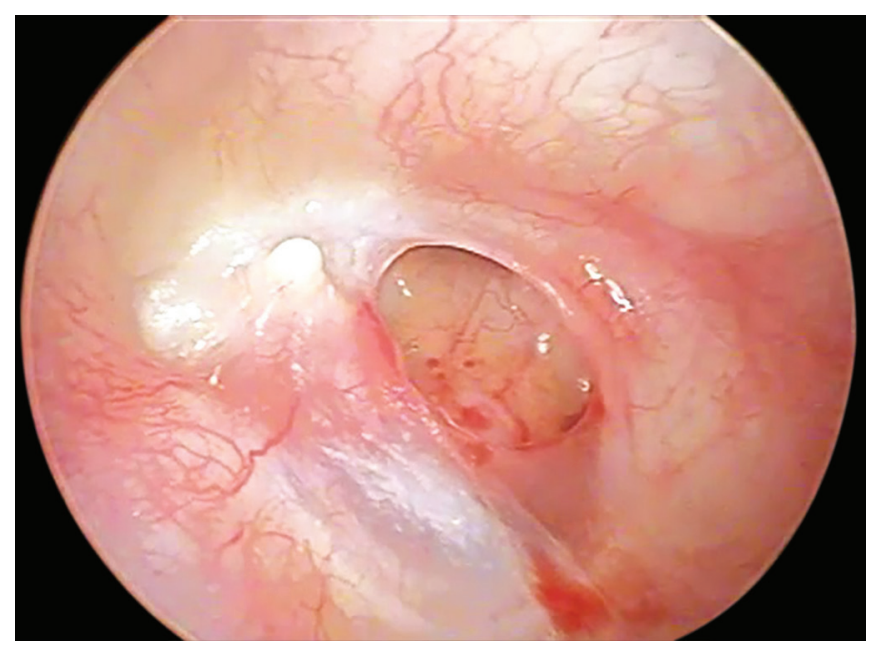

Fig. 2: Endoscopic visualization of the complete tympanic membrane

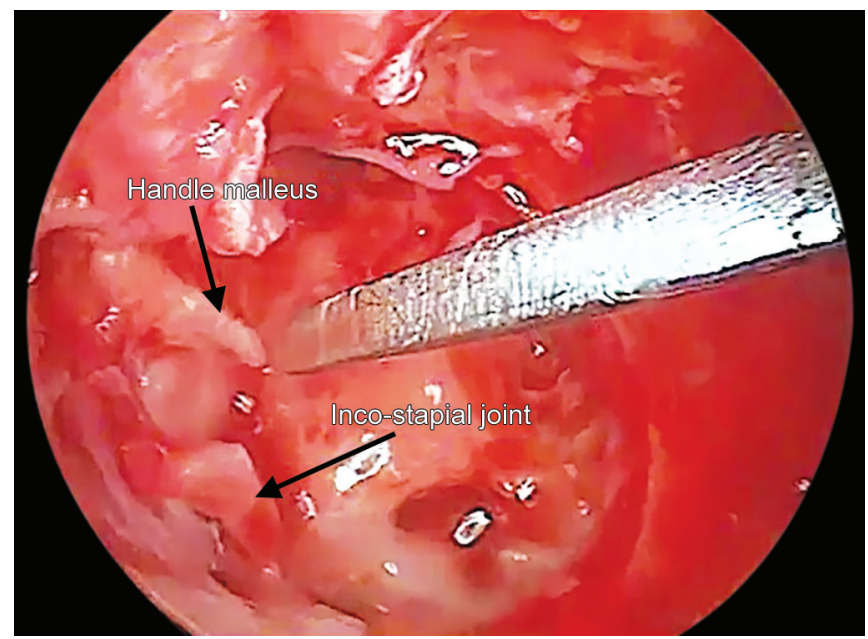

Fig. 3: Middle ear and ossicular chain inspection during endoscopic surgery

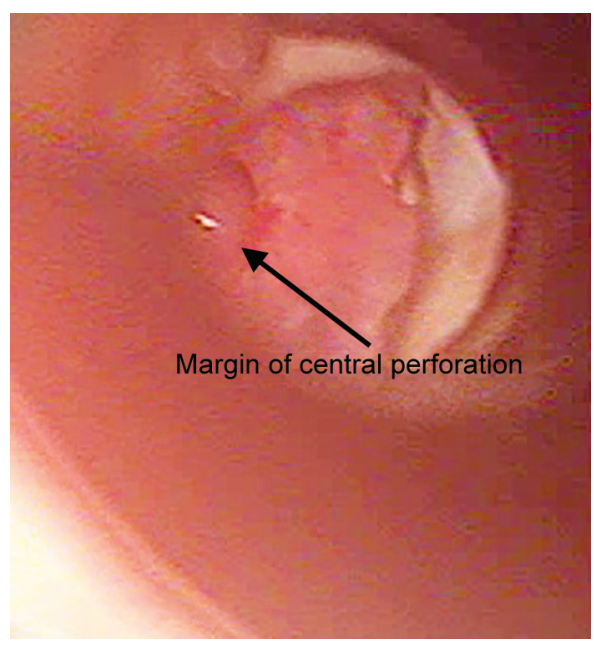

Fig. 4: Limited view with a microscope

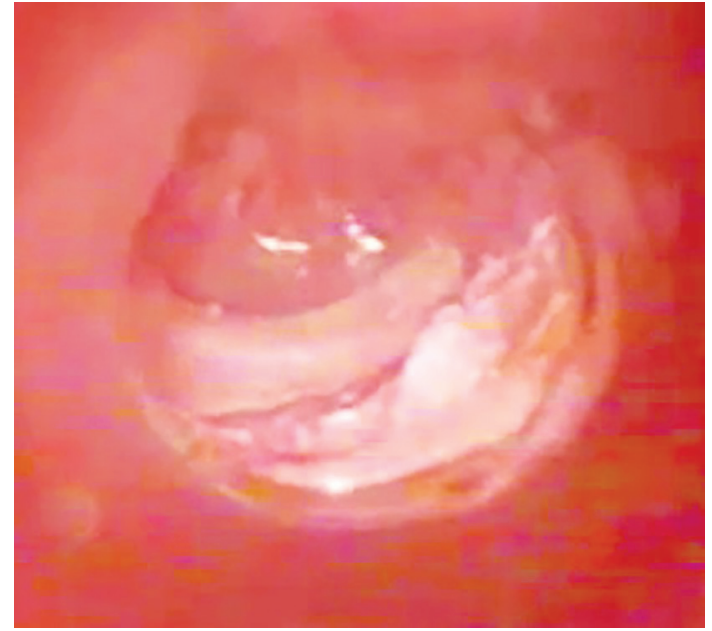

Fig. 5: Middle ear assessment under a microscope

endoscopes were employed to visualize the hidden areas if deemed necessary. Although none of these examinations yielded findings that required us to modify the surgery, we find it to be a valuable step toward the goal of the surgery.

\section{Discussion}

Traditionally, myringoplasty or tympanoplasty type I is performed with an operative microscope. El-Guindy ${ }^{1}$ in 1992 was the first to publish a series on endoscopic myringoplasty although it was Thomassin et al. ${ }^{3}$ who reported the use of endoscopic guided surgery to look at the hidden areas during cholesteatoma surgery since 1987. McKennan ${ }^{2}$ and Youssef ${ }^{8}$ reported the second-look mastoids copy to rule out the residual cholesteatoma.

Endoscopic technique offers a new perspective to understand the anatomy, pathology, and operative techniques. A review of endoscopic ear surgeries demonstrated that there has been an increase in publications in recent years, which reflects acceptance and comfort with the endoscope. Primary indications identified for endoscopic ear surgeries included cholesteatoma removal and myringoplasty. Enough evidence is found in the literature, on the benefit of observational use of the endoscope in ear surgery, and 
the feasibility of endoscope as a microscope replacement has also been studied. ${ }^{9}$

Transcanal tympanoplasty leaves a small or no visible incision, there is little change in ear anatomy, and it can be done in a short time. It is ideal for the repair of tympanic membrane perforations without chronic inflammation or cholesteatoma and/or necrosis of the ossicles.

Comparing endoscopic and microscopic myringoplasty type 1 tympanoplasty based on the success of closure of perforation, some authors had better results with microscope $e^{4,6,10,11}$ and others with endoscope, ${ }^{7,12}$ but these results were statistically insignificant. On comparing the ABG closure, Jyothi et al. ${ }^{6}$ reported a significant difference in favor of microscopic procedures done via postaural route. Other authors ${ }^{5,10,11}$ also reported better ABG closure with a microscope, but Ohki et al. ${ }^{12}$ had better results with the endoscope. In our study, the success rates were only marginally in favor of the endoscopic technique for perforation repair and ABG closure.

Almost all the studies performed transcanal endoscopic and postaural or endaural microscopic surgery, and this is reflected in the time taken for the surgery. Microscopic procedures were thus

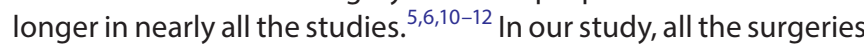
were done via the transcanal route, and the time taken was similar in both procedures. In cases where drilling of canal wall was necessary with the microscope, the surgeries were prolonged. None of the endoscopic procedures required bone removal.

Maran et al. ${ }^{10}$ reported that microscope provided a better result with large perforations. This result was replicated in this or other studies. ${ }^{8,9}$ A meta-analysis on endoscopic versus microscopic type I tympanoplasties by Pap et al. ${ }^{13}$ found surgical outcomes of endoscopic comparable to the microscopic type I tympanoplasty. Moreover, microscopic surgery was associated with a postaural scar and the need for canaloplasty, and these were the areas where the endoscope scored over the microscope.

Microscope provides better depth perception and the freedom to use both hands but gives a linear view through the ear canal and the deep recesses stay hidden. An endoscope on the other hand bypasses the ear canal tunneling effect and provides a much wider view. The rotational movements of angled scopes provide a panoramic view of the hidden areas of the middle ear cleft. Tubal orifice, incudostapedial joint, and oval and round window niches can be seen easily with the endoscope. When the external ear canal is narrow and tortuous and/or anterior canal wall bony overhang is present, canaloplasty needs to be carried in microscopic but not in endoscopic surgery. Microscopes also require adjustment and patient head rotation during surgery, while forward and reverse movements of the endoscope can easily produce close-up and angled images when necessary. With the advent of endoscope holders and high definition systems and factoring in the lower cost of setting up an endoscopic facility with a wider utility in otorhinolaryngology practice, the scope of using an endoscope in ear surgery has increased manifold.

\section{Conclusion}

Endoscopic ear surgery follows the same basic principles as microscopic surgery. The graft uptake rate, hearing improvement, and time taken for surgery with an endoscope are comparable with those of microscopic myringoplasty. The endoscope is valuable in overcoming anatomical obstructions without canaloplasty and in visualizing hidden areas of the middle. At the same time, there is a steeper learning curve, the inconvenience of frequent cleaning of endoscope tip, and only one hand being available for manipulations, which can be eased with the use of an endoscope holder. Considering better visualization via a minimal access approach and equivalent results to microscopic surgery, we recommend the use of an endoscope for type I tympanoplasties, as a primary tool or an accessory tool to the microscope.

\section{ORCID}

Ravneet R Verma $\odot$ https://orcid.org/0000-0002-0007-7855 Ravinder Verma (1) https://orcid.org/0000-0002-3621-2537

\section{References}

1. El-Guindy A. Endoscopic transcanal myringoplasty. J Laryngol Otol 1992;106(6):493-495. DOI: 10.1017/s0022215100119966.

2. McKennan KX. Endoscopic "second look" mastoidoscopy to rule out residual epitympanic/mastoid cholesteatoma. Laryngoscope 1993;103(7):810-814. DOI: 10.1288/00005537-199307000-00016.

3. Thomassin JM, Korchia D, Doris JM. Endoscopic-guided otosurgery in the prevention of residual cholesteatomas. Laryngoscope 1993;103(8):939-943. DOI: 10.1288/00005537-199308000-00021.

4. Harugop AS, Mudhol RS, Godhi RA. A comparative study of endoscope assisted myringoplasty and microscope assisted myringoplasty. Indian J Otolaryngol Head Neck Surg 2008;60(4):298-302. DOI: 10.1007/s12070-008-0099-5.

5. Lade H, Choudhary SR, Vashishth A. Endoscopic vs microscopic myringoplasty: a different perspective. Eur Arch Otorhinolaryngol 2014;271(7):1897-1902. DOI: 10.1007/s00405-013-2673-z.

6. Jyothi AC, Shrikrishna BH, Kulkarni NH, etal. Endoscopic myringoplasty versus microscopic myringoplasty in tubotympanic CSOM: a comparative study of 120 cases. Indian J Otolaryngol Head Neck Surg 2017;69(3):357-362. DOI: 10.1007/s12070-017-1147-9.

7. Plodpai, Y,Paje, N. The outcomes of overlay myringoplasty: endoscopic versus microscopic approach. Am J Otolaryngol 2017;38(5):542-546. DOI: 10.1016/j.amjoto.2017.05.007.

8. Youssef TK, Poe DS. Endoscope-assisted second-stage tympanomastoidectomy. Laryngoscope 1997;107(10):1341-1344. DOI: 10.1097/00005537-199710000-00009.

9. Kozin ED, Gulati S, Kaplan AB, et al. Systematic review of outcomes following observational and operative endoscopic middle ear surgery. Laryngoscope 2015;125(5):1205-1214. DOI: 10.1002/ lary.25048.

10. Maran RK, Jain AK, Karipriya GR, et al. Microscopic versus endoscopic myringoplasty: a comparative study. Indian J Otolaryngol Head Neck Surg 2019;71(Suppl. 2):1287-1291. DOI: 10.1007/s12070-018-1341-4.

11. Dundar R, Kulduk E, Soy FK, et al. Endoscopic versus microscopic approach to type I tympanoplasty in children. Int J Pediatr Otorhinolaryngol 2014;78(7):1084-1089. DOI: 10.1016/j.ijporl. 2014.04.013.

12. Ohki M, Kikuchi S, Tanaka S. Endoscopic type-1 tympanoplasty in chronic otitis media: comparative study with a postauricular microscopic approach. Otolaryngol Head Neck Surg 2019;161(2): 315-323. DOI: 10.1177/0194599819838778.

13. Pap I, Tóth I, Gede N, et al. Endoscopic type I tympanoplasty is as effective as microscopic type I tympanoplasty but less invasive: a metaanalysis. Clin Otolaryngol 2019;44(6):942-953. DOI: 10.1111/coa.13407. 